Available online at:

https://acta-acustica.edpsciences.org

\title{
Investigating the influence of seating area design and enclosure on the seat-dip effect using scale model measurements
}

\author{
Henna Tahvanainen ${ }^{1,}{ }^{*}$, Tapio Lokki ${ }^{1}$, Hyung-Suk Jang ${ }^{2}$, and Jin-Yong Jeon ${ }^{2}$ \\ ${ }^{1}$ Department of Computer Science, Aalto University School of Science, 00076 Aalto, Finland \\ ${ }^{2}$ Architectural Acoustics Laboratory, Hanyang University, Seoul 133-791, South Korea
}

Received 24 February 2020, Accepted 20 August 2020

\begin{abstract}
The low-frequency attenuation in the direct sound due to the concert hall seats, i.e., the seat-dip effect, is studied with the help of a scale model comprising an adjustable seating area and an enclosed box. More particularly, different seat underpass sizes and floor raking angles are studied, and the results are averaged over multiple source positions. With the measurements on the seating area only, the main seat-dip frequency is found to depend on the seat back rest height, and on the degree of obstruction of the seat underpass. The attenuation bandwidth is found to depend mainly on the floor raking. The differences become less clear when seating area is enclosed by concert hall walls and ceiling because the early reflections from the concert hall geometry compensate the low-frequency attenuation in the direct sound. In addition, the low frequencies below the main seat-dip frequency are found to increase in the presence of unobstructed seat underpasses, and such seats are recommended for the maximal bass response in a concert hall.
\end{abstract}

\section{Introduction}

Scale models serve as a great tool to study acoustical properties of complex geometries, in particular at low frequencies. One of the low-frequency phenomena that has been studied with the scale models is the seat-dip effect (SDE) in concert halls (see, e.g. [1-6]). The SDE may be perceptually significant in halls with insufficient reflected sound energy [7], and a threshold of audibility for the main attenuation dip has been obtained using simulated concert halls with relatively short reverberation times [8].

The SDE arises from the complex seating area geometry as a series of diffracted and reflected sound waves that interfere either destructively or constructively with the direct sound, causing attenuation at some frequencies and boosts at other frequencies, respectively. These diffractionreflection phenomena that explain the mechanism of the SDE were confirmed by Ishida $[5,9]$ by using a simplified parallel barrier scale model.

The maximum attenuation of the SDE occurs at low frequencies between 80 and $300 \mathrm{~Hz}$ due to the destructive interference between the direct sound and sound diffracted from the tops of the seat backs that then reflects of the floor or under the seat in case of a seat underpass. The frequency of the maximum attenuation is influenced predominantly by the dimensions of the seats, such as height of the back rest and underpass below the seat $[3,10]$, as well as the angle of arrival of the direct sound [3-5].

\footnotetext{
*Corresponding author: henna.tahvanainen@aalto.fi
}

The two seminal papers published in $1964[1,2]$ that introduced the concept of SDE used scale models to show, among others, that the frequency of the maximum attenuation depends mainly on the seat height. Later, Ishida's measurements [5] covered the aspects of floor raking, underpass and receiver height, angle of incidence, and the effect of stage risers, audience, and the aisle. Ishida found that with a larger vertical incidence angle (represented by a higher source) the main seat-dip frequency increases with decreasing floor raking or underpass height, while with a lower source, the attenuation magnitude increases. Floor raking with constant stage and source height change the vertical angle of incidence of the direct sound. Meanwhile, Davies [4] used a scale model to study the row width, and he showed that the seat-dip effect does not change much beyond the width of ten seats. Later, Davies and Lam [11] concluded that the seat-dip attenuation is influenced by diffraction from seats outside of the direct line of propagation.

While the main seat-dip frequency and the attenuation width have been studied extensively, there is also some indication that the frequencies below the main seat-dip frequency should be included in the SDE discussion. It has been suggested that the seats whose underpasses are not visually obstructed, increase significantly the low frequencies below $100 \mathrm{~Hz}[4,5,10]$.

Altogether, the SDE has mainly been studied from the perspective of a single source-receiver pair, and mostly from the perspective of the frequency response at 15 or $20 \mathrm{~ms}$ after the direct sound. However, the results depends on the specific source-receiver combination, because the SDE 
is sensitive to both vertical and horizontal arrival angle of the direct sound. A recent approach of averaging several sources positioned like a symphony orchestra on stage [12] has revealed a relationship between the seating design and the magnitude response of the SDE in concert halls [10]. Namely, the seats with underpasses on a flat floor yield a seat-dip attenuation extending over a wide frequency band with the maximum attenuation dip at around 150-300 Hz, while seats with underpasses blocked by a step-wise raking floor show narrow band attenuation with a maximum attenuation dip at around $100 \mathrm{~Hz}$ [10].

This paper attempts to further clarify the effect of floor and seat design on the SDE with the help of an acoustic scale model, which allows to study seat dimensions and floor raking separately. In addition, the SDE is studied as a result of an average of several source positions. Measurements are performed with seating area only, and with seating area in an enclosed box approximating a chamber music hall.

\section{Scale model}

The use of scale models in acoustics is based on the assumption that dimensions, absorption properties of the materials, and air attenuation can be scaled with frequency. For example, it is straightforward to show that frequencies and dimensions have a direct scalable relationship, while some additional assumptions about material porosity must be made in order to scale air viscosity [13].

\subsection{Scale model dimensions and materials}

Figure 1 shows the applied 1:10 scale model, which consisted of 336 seats arranged in 24 rows of 14 seats with the distance of first row to stage equal to $1.15 \mathrm{~m}$. The area of the stage was $8 \times 4 \mathrm{~m}$ (height $1 \mathrm{~m}$ ), and the total area of the model was $24 \times 9 \mathrm{~m}$.

The seating configuration was chosen based on observations that at least 10 seats per row, and at least 6 rows are required for the SDE to form [4]. The seating area was designed complying with the guidelines for concert halls provided in Metric Handbook [14] regarding seat height, seat depth, seat inclination, row spacing, and clear way. Each row of seats was glued onto an medium-density fibre (MDF) board of a width corresponding to the row spacing. These boards could then be placed one after the other on the floor, or stacked on a staircase structure in the case of the raked floor.

Three floor settings (flat, moderately raked, highly raked) were measured with three different underpass heights $(0 \mathrm{~cm}, 10 \mathrm{~cm}, 24 \mathrm{~cm})$ for the seats. The height of the seat underpass was varied extending the seat back rest with an additional piece of PVC foam board. In order to apply this extension, the seat rows were taken out of the scale model one by one for modification.

The measurements were completed with unoccupied seats. However, some of the measurements were repeated with occupied seats, as well and they are reported in ref. [15], and are thus not in the scope of this research.
In addition, some of the seating configurations were measured with enclosing the seating area with walls and ceiling. The front and back walls were constructed with removable acrylic plastic windows in order to move the source and receiver inside the scale model. The total volume of the hall was about $2250 \mathrm{~m}^{3}$, and in order to keep the volume constant, the height of the walls and the ceiling were set to $10 \mathrm{~m}$ for the flat floor, and $14.5 \mathrm{~m}$ for the raked floor. The details of the scale model dimensions are listed in Table 1.

The scale model materials are listed together with their random incident absorption coefficients in Table 2. The absorption coefficients of the seats and the audience have been measured in scaled reverberation room during a previous study [16]. Based on the recommendation of that study, the MDF board used on the floor of scale model was varnished with oil-based varnish in three layers in order to best approximate the absorption coefficients of the real concert hall floor. Since the floor and the walls were built specifically for the current study, their absorption coefficients were verified in the scaled reverberation room with the measurement set-up described in the previous study, that complies with the ISO 354 [17].

\subsection{Air attenuation}

Air attenuation generally presents a problem for acoustic scaling because it is more prominent at high frequencies than at low frequencies [18]. Typically, scale model measurements are run at drier-than-usual air conditions or by replacing air with nitrogen to compensate for this effect $[13,16,19]$. Alternatively, filtering can be applied for correcting the air absorption, but it may introduce artifacts (see, e.g. [20]).

However, the air attenuation mechanism is much slower than other attenuation mechanisms below $5 \mathrm{kHz}$ at the relative humidity levels (40-60\%) typically found in concert halls, which means that air absorption can largely be ignored [13]. This means that for the scale model measurements no measures to correct air absorption are needed below $500 \mathrm{~Hz}$, which covers the main SDE frequency. In addition, due to the comparative nature of the results in this paper, it was not seen necessary to take any measures to correct the air absorption throughout the whole frequency range of interest of the SDE up to $1 \mathrm{kHz}$. Consequently, it must be noted that at higher frequencies the scale model overestimates the air absorption by about a factor of 10 , meaning the reverberation time T30 of the enclosed scale model, measured to be $0.6 \mathrm{~s}$, is expected to be shorter than that of the chamber music hall that it approximately corresponds to in real size.

\section{Measurements}

\subsection{Setup}

The measurements with the scale model were carried out in a semi-anechoic room of a volume of $71.4 \mathrm{~m}^{3}$ at Hanyang University, Seoul. The scale model was placed in 
(a)

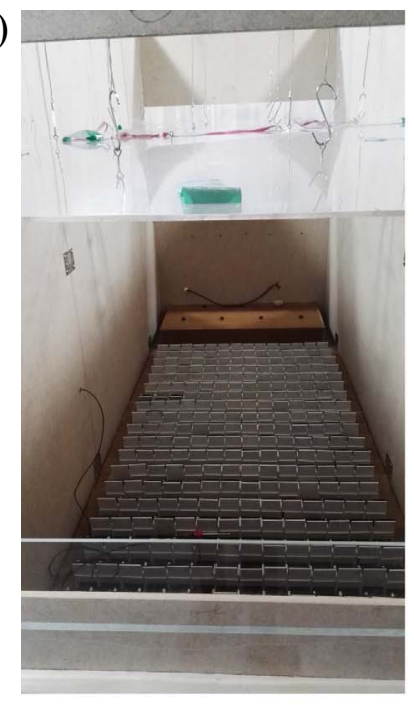

(b)

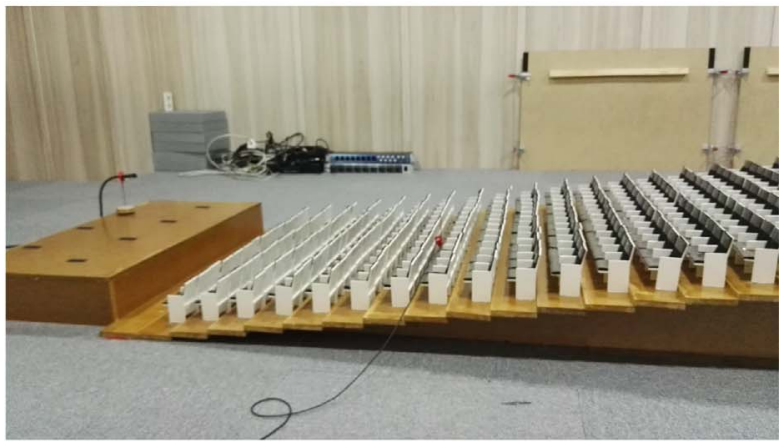

(c)

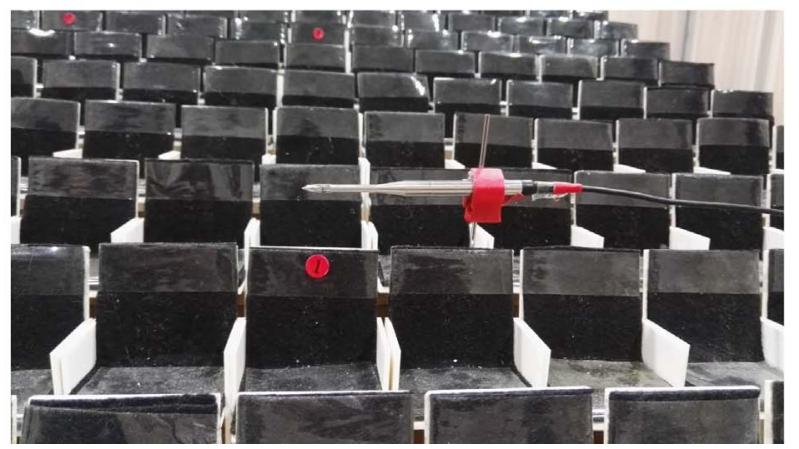

Figure 1. The scale model (a) concert hall box, (b) seating area, and (c) the position of the receiver.

Table 1. The seating dimensions of the scale model scaled to real values.

\begin{tabular}{lc}
\hline Measure & $\mathrm{cm}$ \\
\hline Rowspacing & 80 (and 105) \\
Clearway & 35 \\
Seat height & 88 \\
Seat back rest height & 64 \\
Seat underpass height & Closed, 10, 24 \\
Floor raking step & Flat, 10,24 \\
and corresponding angle & $0^{\circ}, 7^{\circ}, 16^{\circ}$ \\
\hline
\end{tabular}

the room in a way that the closest wall was about $1 \mathrm{~m}$ away from the edge of the scale model, meaning that a closest reflection from the measurement room would arrive at about $60 \mathrm{~ms}$ after the direct sound (in the full model). The measurements were run in the course of several days. During this time, the weighted A-level background noise varied between 17 and $30 \mathrm{~dB}$, room temperature between 19 and $23{ }^{\circ} \mathrm{C}$ and relative humidity between 33 and $44 \%$.

A spark source (BDMSI-040528, China, $15 \mu \mathrm{A}$, distance between electrodes $5 \mathrm{~mm}$ ) was set up at the height of $1.2 \mathrm{~m}$, and seven source locations on the stage were chosen on a grid shown in Figure 2. The source voltage was set to $15 \mathrm{~V}$, which yielded a maximum peak sound pressure level of $101 \mathrm{~dB}$ at $1 \mathrm{~m}$ (real scale). The directivity of the spark source was not measured, but Ayrault et al. [21] have shown that for a 5-mm distance between the electrodes, the spark source is nearly omnidirectional with a maximum deviation of about $-2.5 \mathrm{~dB}$ at 90 and 120 . Equivalently, most musical instruments radiate omnidirectionally at low frequencies [22].

The source height of $1.2 \mathrm{~m}$ was chosen because most musicians on stage are seated holding instruments at about that height. Keeping the source height fixed means that the vertical angle of incidence changes due to floor raking. However, compensation for the change in the vertical angle of incidence by stage height was not considered because the focus of the paper was the overall effect of the seating area.

The receiver was a $1 / 8^{\prime \prime} \mathrm{B} \& \mathrm{~K}$ microphone (Type 4138) connected with a G.R.A.S. type $26 \mathrm{AC}$ connector to a B\&K Nexus microphone preamplifier. The sounds were recorded with a MOTU 896mk2 Hybrid FW at sampling rate of $192 \mathrm{kHz}$ connected to a MacBook Pro 2015 with Adobe Audition software.

The receiver was set at the height of the listeners' ears, at $1.2 \mathrm{~m}$. The receivers were located at $6.7,10.9,15.0$, and $19.2 \mathrm{~m}$ from the stage edge, on the 7 th and 10th seat of the row. These locations were chosen to resemble the previously conducted concert hall measurements [10]. For each sourcereceiver combination three repetitions were recorded, and 
Table 2. The materials and their scaled absorption coefficients used in the scale model.

\begin{tabular}{|c|c|c|c|c|c|c|c|}
\hline \multirow[t]{2}{*}{ Part } & \multirow[t]{2}{*}{ Material (thickness) } & \multicolumn{6}{|c|}{ Absorption coefficients at octave bands $(\mathrm{Hz})$} \\
\hline & & 125 & 250 & 500 & $1 \mathrm{k}$ & $2 \mathrm{k}$ & $4 \mathrm{k}$ \\
\hline Floor & Varnished MDF (12 mm) & 0.05 & 0.10 & 0.14 & 0.14 & 0.15 & 0.17 \\
\hline Seats & PVC foam board ( $2 \mathrm{~mm})$, felt & 0.13 & 0.15 & 0.21 & 0.41 & 0.48 & 0.58 \\
\hline Audience & Unvarnished MDF (12 mm), felt, paper mass & 0.23 & 0.33 & 0.52 & 0.76 & 0.88 & 0.79 \\
\hline Ceiling & Acrylic (5 mm) & & & & & & \\
\hline
\end{tabular}

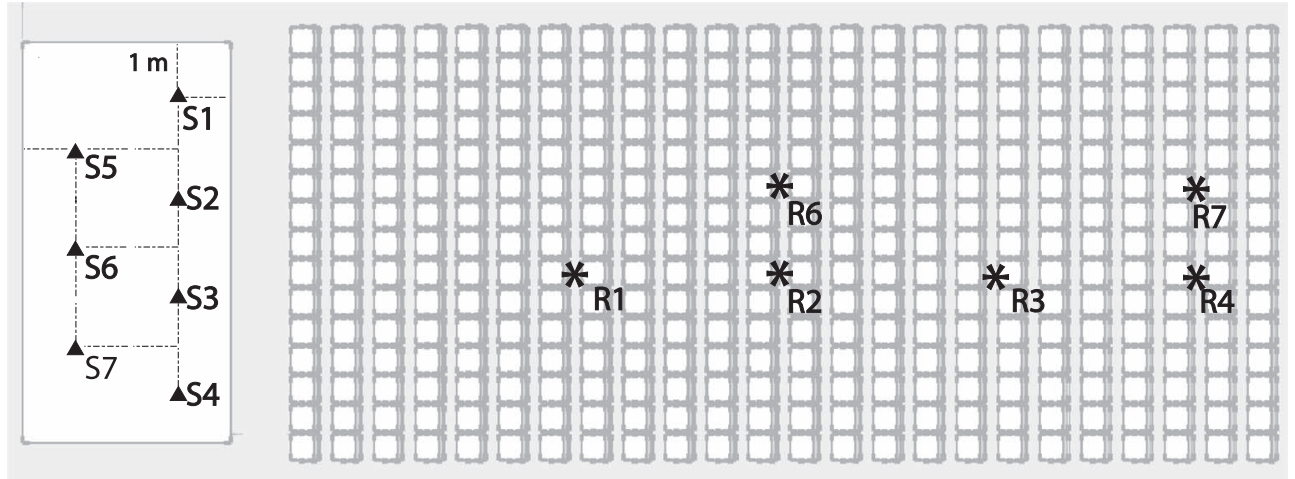

Figure 2. The source-receiver locations of the scale model. The line segment on stage is $1 \mathrm{~m}$.

their frequency responses were averaged and $1 / 3$ octave smoothed in the analysis stage.

A calibration measurement was run at a source-receiver distance of $1 \mathrm{~m}$. In order to separate the direct sound from the reflections of the measurement room surfaces, the source and the receiver were elevated to $1.5 \mathrm{~m}$, and located a distance of $2 \mathrm{~m}$ to the closest wall. The measured calibration frequency response was used to scale the measurements to $10 \mathrm{~m}$ in real scale (corresponding to $G$ parameter). In addition, in the analysis stage, in order to show only the excess attenuation, the measurements were compensated for spherical divergence. Thus, the excess attenuation shown in the subsequent figures contains effect of the SDE, as well as the absorption of materials and air. However, the two latter absorption mechanisms are not selective as the main attenuation dip, and are more or less constant across different seat geometries and floor raking that are compared.

\subsection{Measurement precision}

Since the measurements spanned over several days and the receiver was moved and re-placed due to altering the seat underpass height and floor raking, some imprecision between the measurements was expected to occur. To estimate this, repeated measurements on two consecutive days with two different seating configurations were run while the measurement setup was dismantled between the measurements.
Figure 3 shows the differences between the repeated measurements in all receiver positions, as well as the measured responses as an example at receiver location R3. The results are compensated for spherical divergence and calibration at $10 \mathrm{~m}$.

The results illustrate two cases that have very different tendencies. In the first case of the steeply raked floor, the difference between the repeated measurements lies within $2 \mathrm{~dB}$ up to about $1 \mathrm{kHz}$, above which the error can be up to $8 \mathrm{~dB}$. In the second case with the moderately raked floor, the difference remains steadily below $4 \mathrm{~dB}$, and below $100 \mathrm{~Hz}$, it is even less than $1 \mathrm{~dB}$.

These cases represent very different sources of imprecision. In the first one, the difference curves are similar for all receiver positions, and the largest differences occur at higher frequencies. It is probably related to the differences in temperature and humidity, or accuracy of receiver location. The second case relates to overall level issues at a wider frequency range, such as the sound pressure level of the source, or receiver orientation.

Nevertheless, in both cases the response below and at the main seat-dip frequency is very repeatable. Above that frequency, the shape of the magnitude response is similar, but there can be a difference in level up to $4 \mathrm{~dB}$ until $1 \mathrm{kHz}$. Within this range, any interpretations related to the level of the magnitude responses should be made with care. Above $1 \mathrm{kHz}$, the differences are so large that no meaningful conclusions can be drawn from the measurements. 

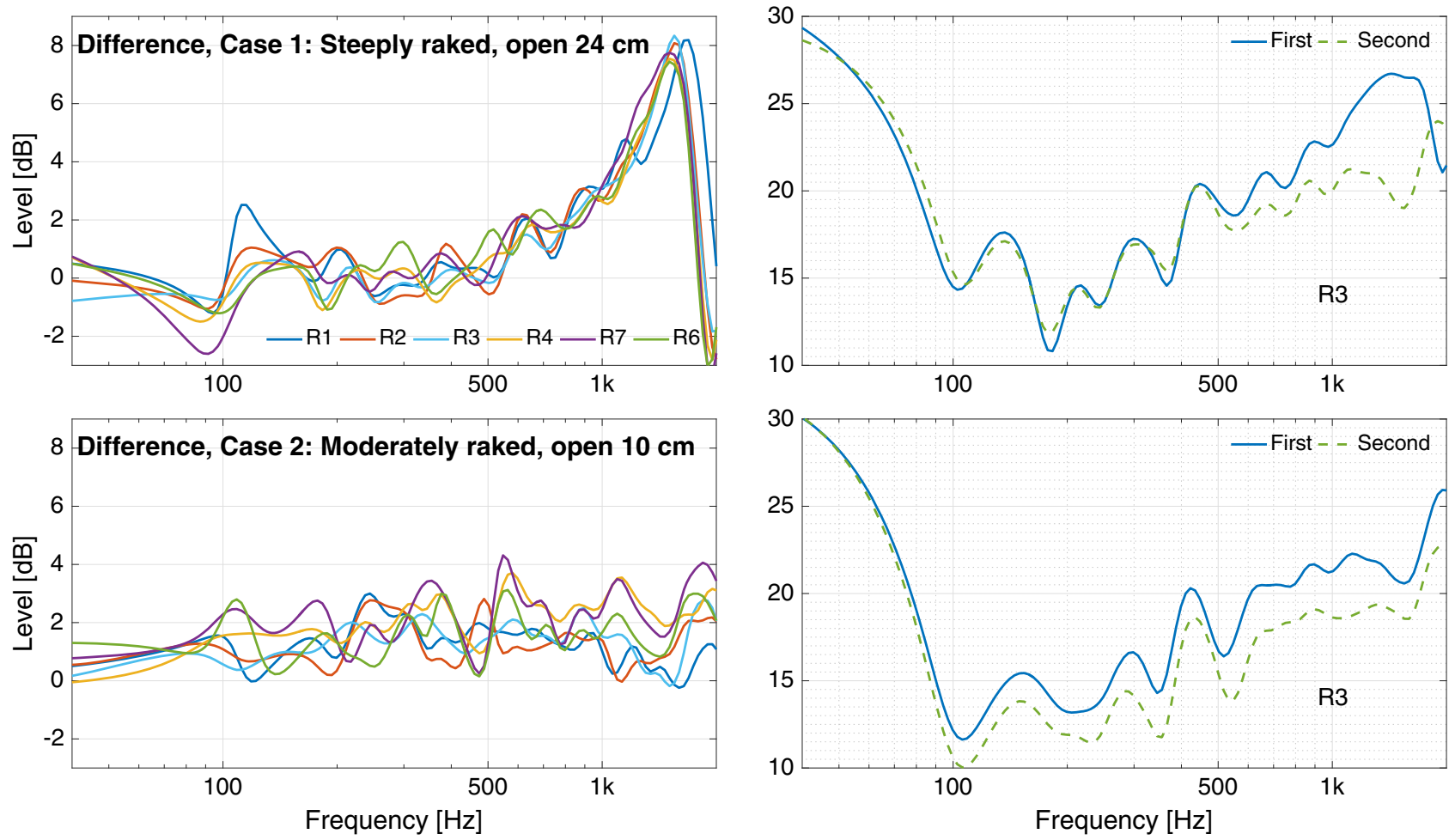

Figure 3. Measurement precision with two different seating cases on two different days referred to as "first" and "second". The left hand side shows the differences between first and second day of measurement for each receiver, while the right hand side shows the averaged responses in one receiver location as an example. The responses are $1 / 3$ octave smoothed.

\section{Results}

The SDE is typically analysed in a time window of 15-20 ms after the direct sound in order to minimise the influence of other reflections in the hall. In the current measurements with the seating area only, the frequency response changes very little after the $15 \mathrm{~ms}$, and the signal level falls below the noise level after about $60 \mathrm{~ms}$. Adding a concert hall around the seating area creates a more complicated series of reflections arriving at the receiver which lengthen the decay time of the signal. In order to compare both measurements, 15-ms analysis window is chosen here. All the analyses are made by averaging the responses from all seven source positions.

\subsection{Seat underpass and floor raking}

Figure 4 shows the frequency responses at $15 \mathrm{~ms}$ after the direct sound for the seat types across floor raking. On the flat and moderately raked floor, the main seat-dip frequency increases with the size of the underpass (in other words, decreases with the seat back rest height), and its amplitude is least severe with the maximal underpass size $(24 \mathrm{~cm})$. Furthermore, the response level below $100 \mathrm{~Hz}$ increases with the size of the underpass.

On the steeply raked floor, however, the responses are very similar across all seat types, especially between closed seats and seats with the $10-\mathrm{cm}$ underpass. This is reasonable as the $10-\mathrm{cm}$ underpass is essentially obstructed, as the underpass is smaller than the step size of the raked floor $(24 \mathrm{~cm})$. In contrast, for the seats with the 24-cm underpass, the underpass and step size are equal. In that case, it seems that some sound is still able to pass under the seats, since the level below $100 \mathrm{~Hz}$, as well as at the main SDE frequency, is higher than in the case of the closed seats with no underpass.

While the response level below and at the main seat-dip frequency seems to depend on the seat type, the responses of all seat types are fairly similar above about $450 \mathrm{~Hz}$ for each floor raking. This is probably because the high frequencies are affected mainly by seat absorption, and diffraction from the tops of the seat backs, both of which are assumed to stay constant with changing underpass size.

In order to study the effect of floor inclination on the SDE, the frequency responses are regrouped in Figure 5. It must be noted that a part of the effects observed here might be attributed to the change in the vertical angle of incidence, albeit by only a few degrees, as angle of incidence increases with floor inclination when the stage and source height are kept constant.

For the closed seats, it can now be seen that the main seat-dip frequency and its level are nearly the same across different floor raking. Some differences appear at the farther receiver positions ( $\mathrm{R} 3, \mathrm{R} 4, \mathrm{R} 7)$.

For the open seats with underpasses, the main seat-dip frequency and level depends on whether the underpass is obstructed or not. For example, the seats with 10-cm underpass behave more similarly on raked floors, the underpasses 

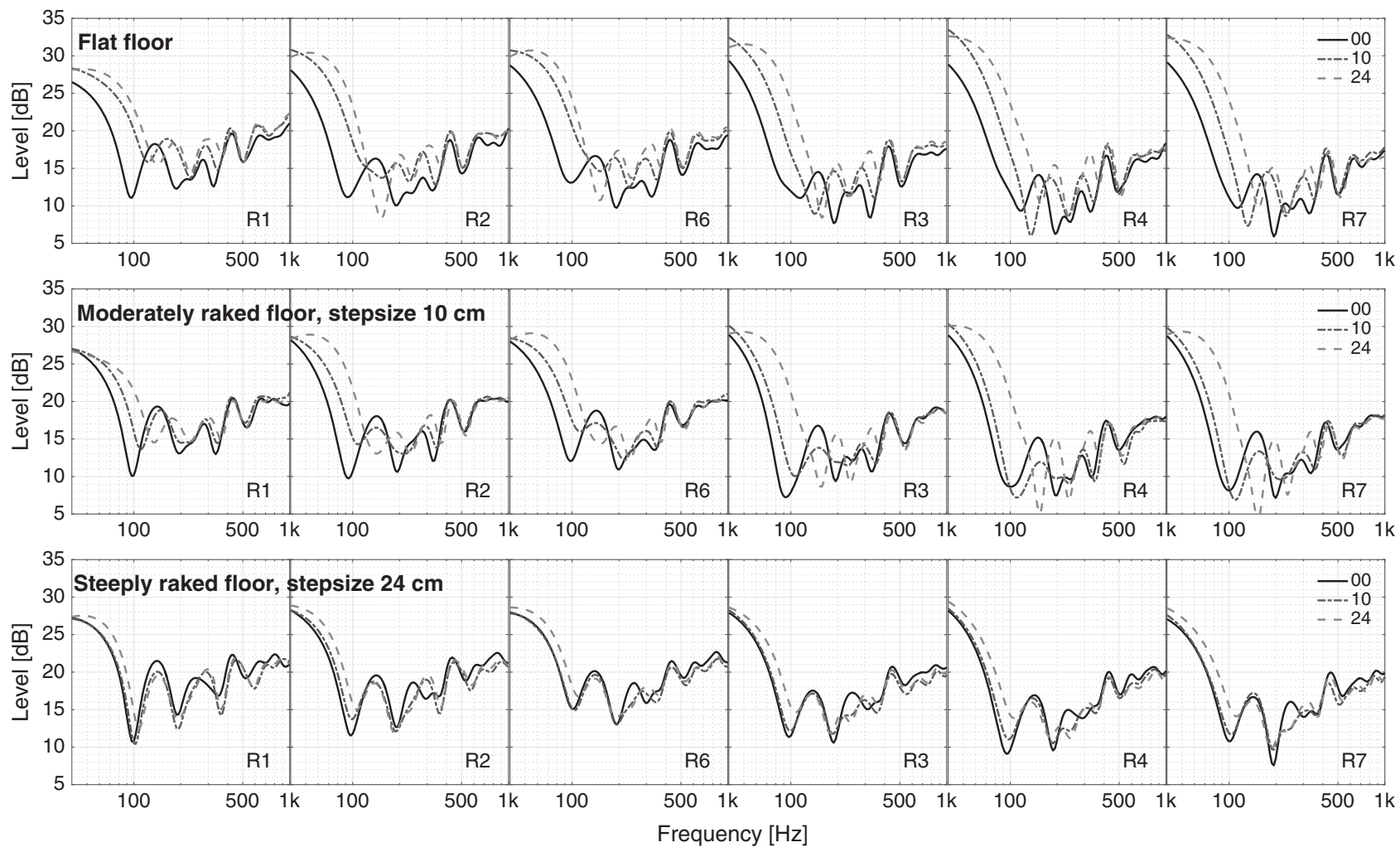

Figure 4. The $1 / 3$-octave smoothed magnitude responses at $15 \mathrm{~ms}$ after the direct sound after calibration at $10 \mathrm{~m}$ and compensation for spherical divergence. The responses show the effect of the seat type across floor raking (closed $=00,10-\mathrm{cm}$ opening $=10$, and $24-$ $\mathrm{cm}$ opening $=24)$.

are obstructed by the raking steps $(10 \mathrm{~cm}$, and $24 \mathrm{~cm})$. In contrast, the seats with the 24-cm underpass behave more similarly on flat floor and on the moderately raked floor, since the underpasses are not fully obstructed.

The measurements show that the seats with underpasses that are not obstructed by the steps have an increased level below $100 \mathrm{~Hz}$. In particular, the results with the seats of 24-cm underpass in the bottom of Figure 5 show that the level of bass reduces with increasing obstruction of the seats. In general, the bass response appears as a wide peak, whose magnitude increases with distance.

For all seat types, the level above $200 \mathrm{~Hz}$ increases with floor raking, and the effect is more pronounced for the closed seats. Apart from measurement uncertainties, this is likely caused by the change of the vertical angle of incidence, as well as a floor reflection, a change in the diffraction from the tops of the seat backs in front of the receiver, and the reflections from the tops of the seats behind the receiver. This could explain the narrow attenuation bandwidth observed in the measurements with a larger set of sources in the actual concert halls with raked floor and closed seats [10].

\subsection{Enclosed box}

Regarding the effect of an enclosed box on the SDE, Figure 6 shows the 15-ms magnitude responses of the scale model with and without the box. It can be seen that the overall level of the responses generally increases when the box is added. The only exception to the case occurs with open seats on a flat floor, where almost no increase in the low frequencies is observed. This suggests that the case of the flat floor and open seats already enables maximal amount of bass, while in the other cases the additional reflections from the concert hall geometry provide the missing low frequencies.

Because of the enclosure, it is also possible to observe the late response of the scale model, and Figure 7 shows the magnitude responses in the enclosed box for 15-ms and 500-ms after the direct sound with different floor and seat types. A few details are very prominent in the 15 -ms magnitude responses. Firstly, looking at the first two rows of subfigures, the raked floor with both seat types has a higher level above $100 \mathrm{~Hz}$ than the flat floor. Secondly, looking at the last two rows of subfigures, the seat type does not make a big difference on a raked floor, but on a flat floor the open seats have the most bass below $200 \mathrm{~Hz}$ while the closed seats feature the steepest seat-dip attenuation. These observations are similar to ones made in the previous section without the enclosed box.

As for the 500-ms responses, the closed seats on the flat floor have a reduced level below $100 \mathrm{~Hz}$, but all the other cases are more equal. In addition, at the farther receiver positions (R3, R4, R7), there is a dip at about $100 \mathrm{~Hz}$, whose amplitude is $2-6 \mathrm{~dB}$ steeper for all the seats that are closed or blocked than for the open seats. A similar 

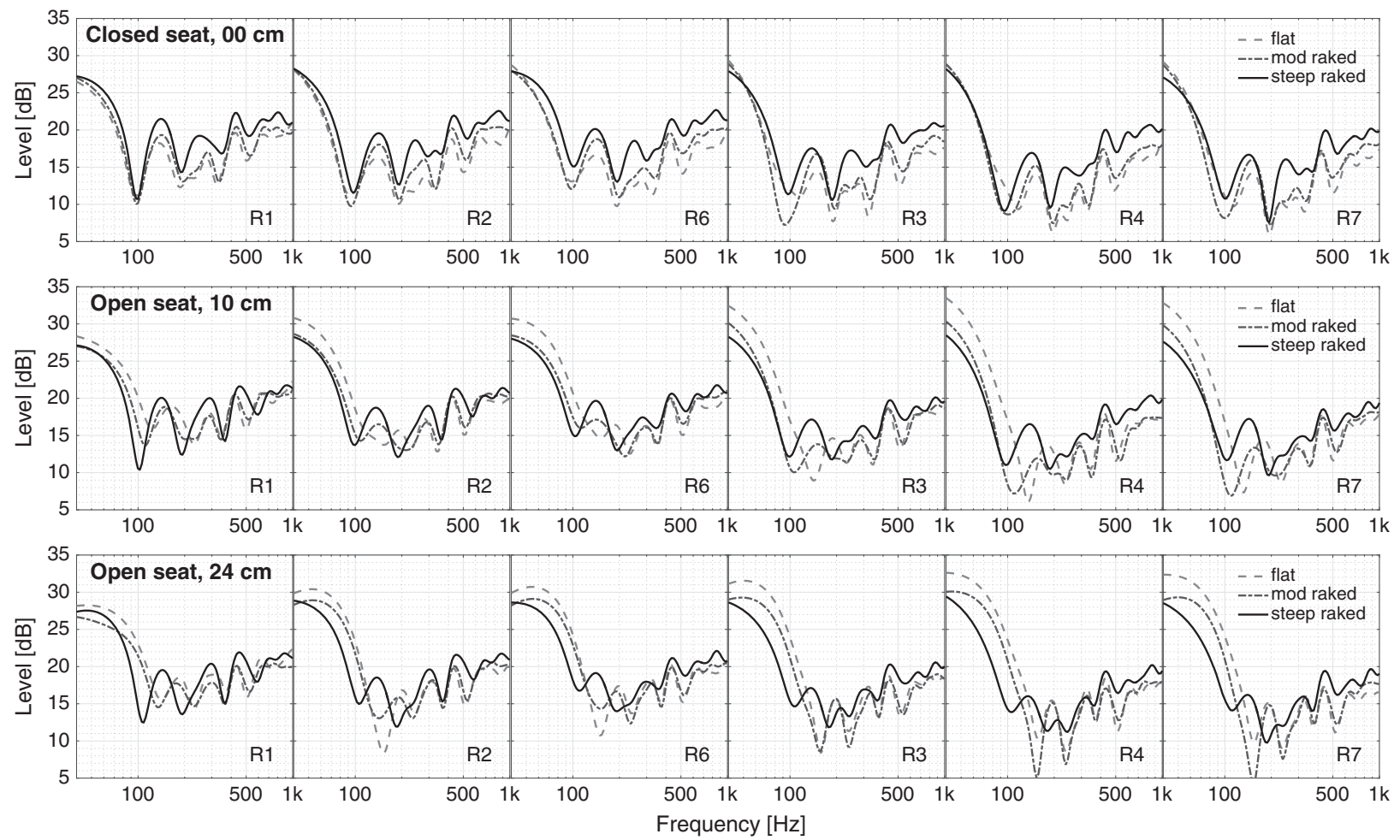

Figure 5. The $1 / 3$-octave smoothed magnitude responses at $15 \mathrm{~ms}$ after the direct sound after calibration at $10 \mathrm{~m}$ and compensation for spherical divergence. The responses show the effect of floor raking across seat types.

dip was observed in the actual concert hall measurements [10], and based on the scale model measurements here, its cause can be linked to the seat underpass.

\section{Discussion}

These scale model measurements aimed at understanding the effect of seat design and floor raking on the frequency and width of the main attenuation dip caused by the SDE.

As for the main dip frequency, previous findings show that the main seat-dip frequency depends on the seat back rest height. The attenuation is expected to dip at a frequency corresponding to about $1 / 4$ wavelength of the seat back rest height [3-5]. In fact, the idea was to consider an effective seat height depending on how much of the seat back rest is blocked by the step-wise raking floor, but because the actual sound path is three-dimensional, the main seat-dip frequency differs slightly from this "theoretical" value in measured concert halls [10].

The results in the current work support these previous findings to a certain degree. The main seat-dip frequency seems to depend on the effective (unobstructed) seat back rest height rather than the floor raking, but the measured and the calculated main seat dip frequency do not always match, as can be seen in Table 3. For example, on the flat floor, the measured frequencies tend to be about $10-20 \mathrm{~Hz}$ higher than the calculated ones, while on the raked floor, the measured frequencies are lower than the calculated ones. In essence, on the flat floor the effective height is shorter, and on the raked floor longer than the physical one. Between floor types, it must also be noted that the vertical angle of incidence is different, and generally the main seat-dip frequency is lower on the raked floors due to the increased vertical angle of incidence.

Another example of three dimensional pathway comes from the results presented by Bradley [3], which imply that if the vertical angle of incidence is small, the horizontal pathway defined by the row spacing may become important. As the dimensions of both the row spacing and seat back rest height are very similar in typical concert hall settings, it means the destructive interference associated with each dimension would cause attenuation at almost the same frequency. In this scale model, the row spacing is $80 \mathrm{~cm}$, while the seat back rest height varies between 64 and $88 \mathrm{~cm}$, being closest to the row spacing on moderately raked floor and open seats with 10-cm underpass. This could explain why the main seat-dip attenuation is at its steepest with seats on moderately raked floor at the farther receiver positions R3-R7 in Figure 5.

In addition, the effect of obstructing the underpass can also been seen in the scale model measurements in Figure 4 on the steeply raked floor. There the frequency of the main attenuation dip is almost constant across all seat types because the step size of $24 \mathrm{~cm}$ covers the underpasses in all seat types, and leaves $64 \mathrm{~cm}$ of the seat back rest in front of the receiver with no underpass.

As for the attenuation width, the scale model measurements here show that floor raking probably the main 


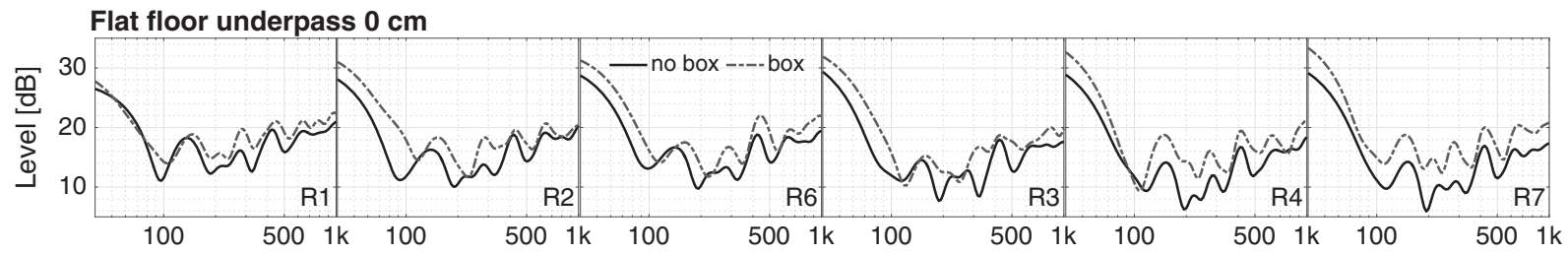

Flat floor, underpass $24 \mathrm{~cm}$

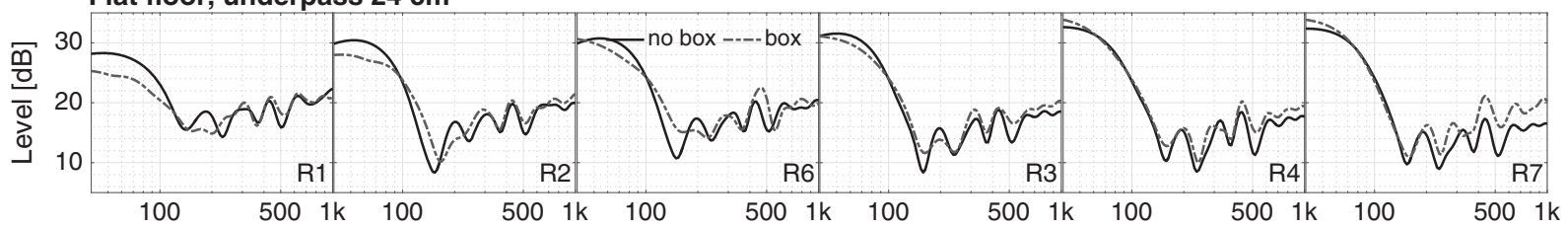

Steeply raked floor, underpass $0 \mathrm{~cm}$

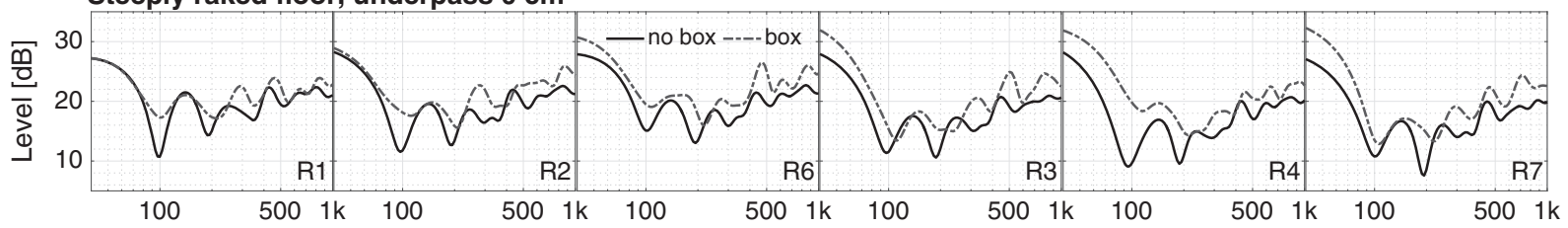

Steeply raked floor, underpass $24 \mathrm{~cm}$

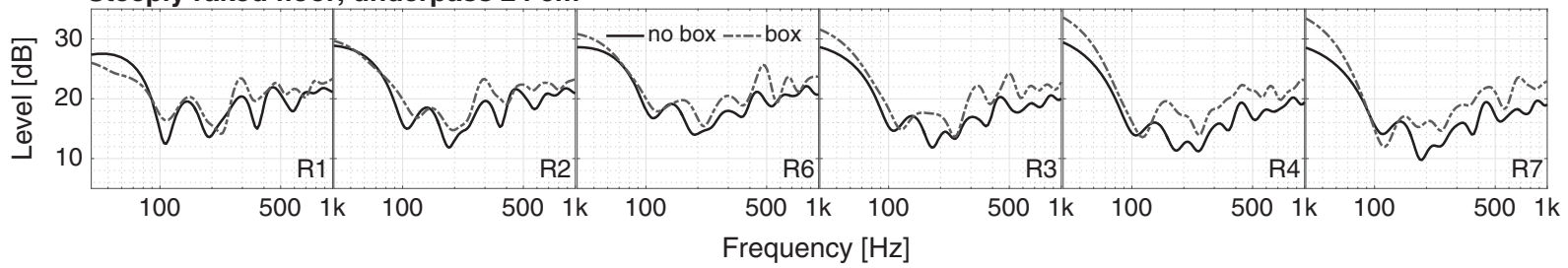

Figure 6. The effect of adding the concert hall geometry at $15 \mathrm{~ms}$ after the direct sound. The responses are $1 / 3$ octave smoothed.

contributing factor to the attenuation width, as steeper raking shows higher levels above about $450 \mathrm{~Hz}$. This can be understood as an increase in positive diffraction coming from the seat tops both in front, and behind the receiver. In addition, there is a floor reflection from the stage in the case of raked floors. It is also possible that the destructive interference between the direct sound and the horizontal pathway become less dominant because of increased angle of incidence, as described by Bradley [3].

Finally, these scale model measurements present an additional discovery that was previously speculated by both Davies [4] and Ishida [5]. Namely, the high response level below $100 \mathrm{~Hz}$, or the bass boost, is associated with the existence of a seat underpass that is not obstructed, and the boost can be as much as $6 \mathrm{~dB}$.

As for the mechanism of the bass boost, already Schultz and Watters [2] noticed a high boost at $60 \mathrm{~Hz}$ (full scale) in their scale model measurements with seats, and attributed it to surface waves, and cylindrical, rather than spherical, wave propagation. Davies [4] and Ishida [5] suspected that it is caused by reflections between the floor and the lower part of the seats, and that several successive reflections from the seating area improve the bass level while worsening the main seat-dip attenuation. However, the results presented here as an average of several source-receiver pairs show that while the bass boost for an underpass always exists, but it does not always lead to the steepest attenuation at the main
SDE frequency. All the measurements were performed with unoccupied seats, yet there is some indication that the presence of audience may partially counteract the bass boost if the underpass is fairly small and can be obstructed by the feet [15].

Consequently, the results suggest that the design of the seats and seating area does not only cover the main SDE frequency and the extension of the attenuation bandwidth up to $1 \mathrm{kHz}$, but also the frequency range below $100 \mathrm{~Hz}$. In practice, the seats with underpasses should be preferred in all cases for maximal level of bass below $100 \mathrm{~Hz}$. For this purpose, an unobstructed seat underpass should be left on raked floors either by adjusting the step size or creating a stepless rake design. It is worth noting that, this suggestion is contrary to the idea presented in previous literature about improving the early bass level by eliminating the seat underpass $[1,4,5]$.

This is an important consideration in particular in concert halls with less reflected energy. Shoebox-shaped halls tend to provide sufficient reflected energy to compensate for the SDE, both perceptually and objectively, while this may not be the case for other hall types [7, 10]. In fact, in these scale model measurements with the shoebox-shaped hall, the reflections from the box reduce the differences between the seating area designs. Such reflections must arrive at non-grazing angles from the ceiling and walls [10]. Particularly in the case of the raked floor, the ceiling 

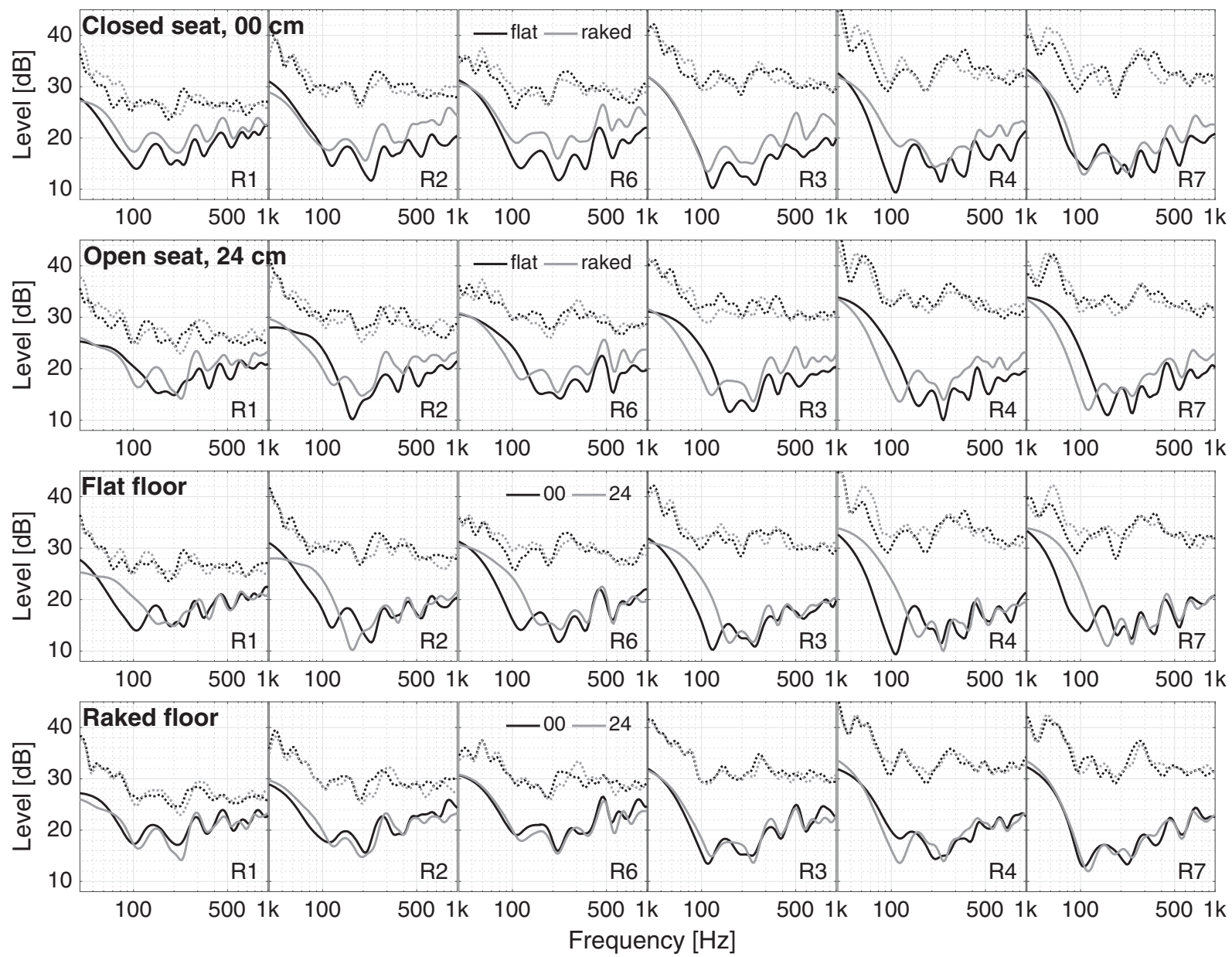

Figure 7. The $15 \mathrm{~ms}$ (solid) and $500 \mathrm{~ms}$ (dotted) magnitude responses of the different seat and floor types measured with the box. The responses are $1 / 3$ octave smoothed.

Table 3. The seat and floor types with the estimated effective seat back rest heights ${ }^{*}$ (the unobstructed part of the seat back rest) together with the calculated main dip frequencies ${ }^{* *}$ with effective seat backrest corresponding to $\lambda / 4$, as well as the measured main attenuation dip frequencies ${ }^{* * *}$ for the floor and seat combinations.

\begin{tabular}{|c|c|c|c|c|}
\hline Floor type & Seat type (underpass) & Eff. back rest* $(\mathrm{cm})$ & SDE calc. ${ }^{* *}(\mathrm{~Hz})$ & SDE meas. ${ }^{* * *}(\mathrm{~Hz})$ \\
\hline Flat & Closed $(0 \mathrm{~cm})$ & 88 & 98 & $94-110$ \\
\hline$"$ & Open $(10 \mathrm{~cm})$ & 78 & 111 & $122-149$ \\
\hline , & Open $(24 \mathrm{~cm})$ & 64 & 135 & $149-158$ \\
\hline Mod. raked & Closed $(0 \mathrm{~cm})$ & 78 & 111 & $94-99$ \\
\hline , & Open $(10 \mathrm{~cm})$ & 78 & 111 & $109-111$ \\
\hline , & Open $(24 \mathrm{~cm})$ & 64 & 135 & $145-158$ \\
\hline Steeply raked & Closed $(0 \mathrm{~cm})$ & 64 & 135 & $97-102$ \\
\hline , & Open $(10 \mathrm{~cm})$ & 64 & 135 & $97-102$ \\
\hline$"$ & Open $(24 \mathrm{~cm})$ & 64 & 135 & $109-115$ \\
\hline
\end{tabular}

becomes closer to the receiver with distance, and thus the associated first reflection arrives earlier. This is another contributing factor to the increased level at high frequencies for raked floor.

Furthermore, the increased bass level could be important for the perception of dynamics in concert halls [23]. The rationale behind this is that the equal loudness contours are significantly closer together at low frequencies and at high sound pressure levels reached by the orchestra at fortissimo. The combined effect leads to loudness differences of up to at least $5 \mathrm{~dB}$ below $100 \mathrm{~Hz}$ in two studied concert halls [23]. While the seating area design seems to have a minor impact on the final frequency response in a fixed concert hall geometry in these scale model measurements, in real concert halls with less reflected energy, the perception of dynamics might be enhanced with unobstructed seat underpasses. 


\section{Conclusion}

In previous concert hall measurements [10], it was observed that the open seats on a flat floor generate a seat-dip effect (SDE) with a wide attenuation bandwidth that is not as severe as the narrow dip generated by the closed seats on a raked floor. Studying the effect of the seat type and the floor raking separately with the scale model reveals some general trends about the SDE.

The results confirm that the unobstructed seat back rest height is the determining factor for the main seat-dip frequency. Furthermore, the results suggest that floor raking is responsible for the decreased attenuation bandwidth. There are several contributing factors to this since by increasing floor raking, 1) the angle of incidence for the direct sound changes, 2) the diffraction from the tops of the seat backs in front and behind the receiver increase, and 3 ) in addition there is a floor reflection and a ceiling reflection within the observed time window of $15 \mathrm{~ms}$. These reflections help level off the SDE with time.

Finally, the results show that the SDE also extends below the main seat-dip frequency. Seats with underpasses boost frequencies below $100 \mathrm{~Hz}$ compared to closed seats whose seat back rest extend all the way to the floor, as hypothesized by [4, 5]. Meanwhile, stepwise raking floor obstructs the seat underpass and reduces the level below $100 \mathrm{~Hz}$. Ensuring adequate seat underpass could be significant for the perception of dynamics [23].

\section{Acknowledgments}

H.T.'s work was supported by Emil Aaltonen Foundation, Finnish Foundation for Technology Promotion, and KAUTE foundation. H.T.'s research visit to Hanyang University was supported by the Foundation for Aalto University Science and Technology. The authors would like to thank Ms. Na-Kyung Yang for her help in building the scale model and in conducting the measurements.

\section{Conflict of interest}

The authors declare that they do not have any conflict of interest.

\section{References}

1. G. Sessler, J. West: Sound transmission over theatre seats. Journal of the Acoustical Society of America 36, 9 (1964) $1725-1732$.

2. T. Schultz, B. Watters: Propagation of sound across audience seating. Journal of the Acoustical Society of America 36, 5 (1964) 885-896.

3. J. Bradley: Some further investigations of the seat dip effect, Journal of the Acoustical Society of America 90, 1 (1991) $324-333$.
4. W. Davies: The Effects of Seating on the Acoustics of Auditoria. PhD Thesis, University of Salford, England, 1992.

5. K. Ishida: The Measurement and Prediction of Sound Transmission over Auditorium Seats. $\mathrm{PhD}$ Thesis, University of Cambridge, England, 1993.

6. D. Takahashi: Seat dip effect: The phenomena and mechanism. Journal of the Acoustical Society of America 1023 (1997) 1326-1334.

7.H. Tahvanainen, A. Haapaniemi, T. Lokki: Perceptual significance of seat-dip related direct sound colorations in concert halls. Journal of the Acoustical Society of America 141, 3 (2017) 1560-1570.

8. W. Davies, T. Cox, Y. Lam, Subjective perception of seat dip attenuation, Acta Acustica United With Acustica 82 (1996) 784-792.

9. K. Ishida: Investigation of the fundamental mechanism of the seat-dip effect - Using measurements on a parallel barrier scale model. Journal of the Acoustical Society of Japan (E) 16, 2 (1995) 105-114.

10. H. Tahvanainen, J. Pätynen, T. Lokki: Analysis of the seatdip effect in twelve European concert halls. Acta Acustica United With Acustica 101, 4 (2015) 731-742.

11. W. Davies, Y. Lam: New attributes of seat dip attenuation. Applied Acoustics 41, 1 (1994) 1-23.

12. J. Pätynen, S. Tervo, T. Lokki, Analysis of concert hall acoustics via visualizations of time-frequency and spatiotemporal responses. Journal of the Acoustical Society of America 133, 2 (2013) 842-857.

13. R. Emori, D. Schuring: Scale models in Engineering Fundamental and Applications. Pergamon Press, 1977.

14. I. Appleton, S. Fischer: Auditoria, ch. 15, in: Metric Handbook, 5th ed. P. Buxton, Editor. Routledge, 2015.

15. H. Tahvanainen, T. Lokki: About the seat-dip effect in the presence of audience, in: Proceedings of the International Congress of Sound and Vibration, Hiroshima, Japan, Jul. 9-12, 2018.

16. J.Y. Jeon, J.K. Ryu, Y.H. Kim, S.-I. Sato: Influence of absorption properties of materials on the accuracy of simulated acoustical measures in 1:10 scale model test. Applied Acoustics 70, 4 (2009) 615-625.

17. ISO 354:2003: Acoustics - Measurement of sound absorption in a reverberation room. International Organization for Standardization, 2003.

18. M. Barron: Auditorium Acoustics and Architectural Design. 2nd ed., Spon Press, New York, USA, 2009.

19. K. Baruch, A. Majchrzak, B. Przysucha, A. Szelkg, T. Kamisinski: The effect of changes in atmospheric conditions on the measured sound absorption coefficients of materials for scale model tests. Applied Acoustics 141 (2018) 250-260.

20. J. Polack, A. Marshall, G. Dodd: Digital evaluation of the acoustics of small models: The midas package. The Journal of the Acoustical Society of America 85, 1 (1989) 185-193.

21. C. Ayrault, P. Béquin, S. Baudin: Characteristics of a spark discharge as an adjustable acoustic source for scale model measurements, in: Acoustics. Nantes, France, 2012.

22. J. Pätynen, T. Lokki: Directivities of symphony orchestra instruments. Acta Acustica United With Acustica 96, 1 (2010) 138-167.

23. T. Lokki, J. Pätynen: Objective analysis of the dynamic responsiveness of concert halls. Acoustical Science and Technology 41, 1 (2020) 253-259. 